\title{
Prosthetic Rehabilitation of Maxillectomy Defects, with Single-Piece Open-Hollow Bulb Definitive Obturator
}

\author{
Ananya Mishra ${ }^{1}$, Kasim Mohamed ${ }^{2}$, Prasanna Kumar ${ }^{3}$, Sathish Kumar Jayagandhi ${ }^{4}$ \\ 1,2 Department of Prosthodontics, Crown and Bridge, Sri Ramachandra Institute of Higher Education and \\ Research (DU), Chennai, Tamilnadu, India. ${ }^{3,4}$ Department of Head and Neck Surgery (ENT), Sri \\ Ramachandra Institute of Higher Education and Research (DU), Chennai, India.
}

\section{INTRODUCTION}

Patients who undergo surgical resection of the maxillo-mandibular structures as a result of trauma, infection or malignancy, suffer from psycho-social setbacks which has a profound impact on their over-all quality of life.1,2 These defects, especially those following maxillectomy, result in oroantral communication, facial deformation, impaired speech and difficulty in deglutition. For the rehabilitation of patients with such defects, surgical and prosthetic treatment options are available. As, not all patients can be successfully rehabilitated with reconstructive surgeries due to postoperative complications like graft rejection, the extent of the surgical defect and high psychological impact factor associated with repeated surgeries, prosthetic rehabilitation proves to be an alternative treatment option. The prosthetic rehabilitation of such patients is challenging as it requires restoration of the lost form, function and aesthetics, under constantly changing state of post-surgical intraoral tissues, with limited mouth opening.

The maxillofacial prosthesis designed to close congenital or an acquired tissue opening, primarily of the hard palate, is known as an obturator. ${ }^{3}$ The obturator has two functional components, one seals the surgical defect and the other replaces the lost dentoalveolarstructures. ${ }^{4-7}$ The design of an obturator may vary depending on the extent of the defect, remnant dentoalveolar complex, soft tissue undercuts and existent muscle physiology.8,9 Among the two designs, solid and hollow, hollow obturators are widely used. The bulb portion of the hollow obturator, which accommodates the surgical defect, can be open or closed ${ }^{9,10}$ and its selection depends on the prosthodontist's clinical decision-making skills and the ease of fabrication. In this article we have discussed the rehabilitated patients with single-piece, openhollow bulb definitive obturator.

Patients undergo extensive maxillary surgical resections due to aggressive lesions like malignancies and deep fungal infections. Prosthetic rehabilitation of such patients with an obturator becomes of paramount importance as it separates the oropharynx from the nasopharynx, reduces the risk of recurrent infections, replaces lost dentoalveolar structures, permits intelligible speech, reinstates mastication and deglutition, restores facial contour and patient's self-esteem. The bulb portion of the obturator extends into the defect and accommodates it, forming a hermetic seal. In this clinical report, we highlight the success of prosthetic rehabilitation of maxillectomy patients using single-piece, open-hollow bulb definitive obturator. The meticulous follow-up carried out reveals the success of the prosthesis and adds practice-based evidence to the maxillectomy rehabilitation outcome.
Corresponding Author: Dr. Kasim Mohamed,

Department of Prosthodontics, Crown and Bridge,

Department No. 8, Third Floor, Faculty of Dental Sciences,

Sri Ramachandra Institute of Higher Education and Research,

Chennai - 600116, Tamilnadu, India.

E-mail: mohamedkasim9@yahoo.com

DOI: $10.14260 / j e m d s / 2021 / 248$

How to Cite This Article:

Mishra A, Mohamed K, Kumar P, et al. Prosthetic rehabilitation of maxillectomy defects, with single-piece open-hollow bulb definitive obturator. J Evolution Med Dent Sci 2021;10(16):1169-1173, DOI: 10.14260/jemds/2021/248

Submission 10-12-2020,

Peer Review 19-02-2021,

Acceptance 26-02-2021,

Published 19-04-2021.

Copyright $(2021$ Ananya Mishra et al. This is an open access article distributed under Creative Commons Attribution License [Attribution 4.0 International (CC BY 4.0)] 


\section{PRESENTATION OF CASE 1}

A 40-year-old female patient underwent left total maxillectomy 3 years ago, following which she developed an unintelligible speech, unaesthetic facial profile and difficulty during oral feeding.
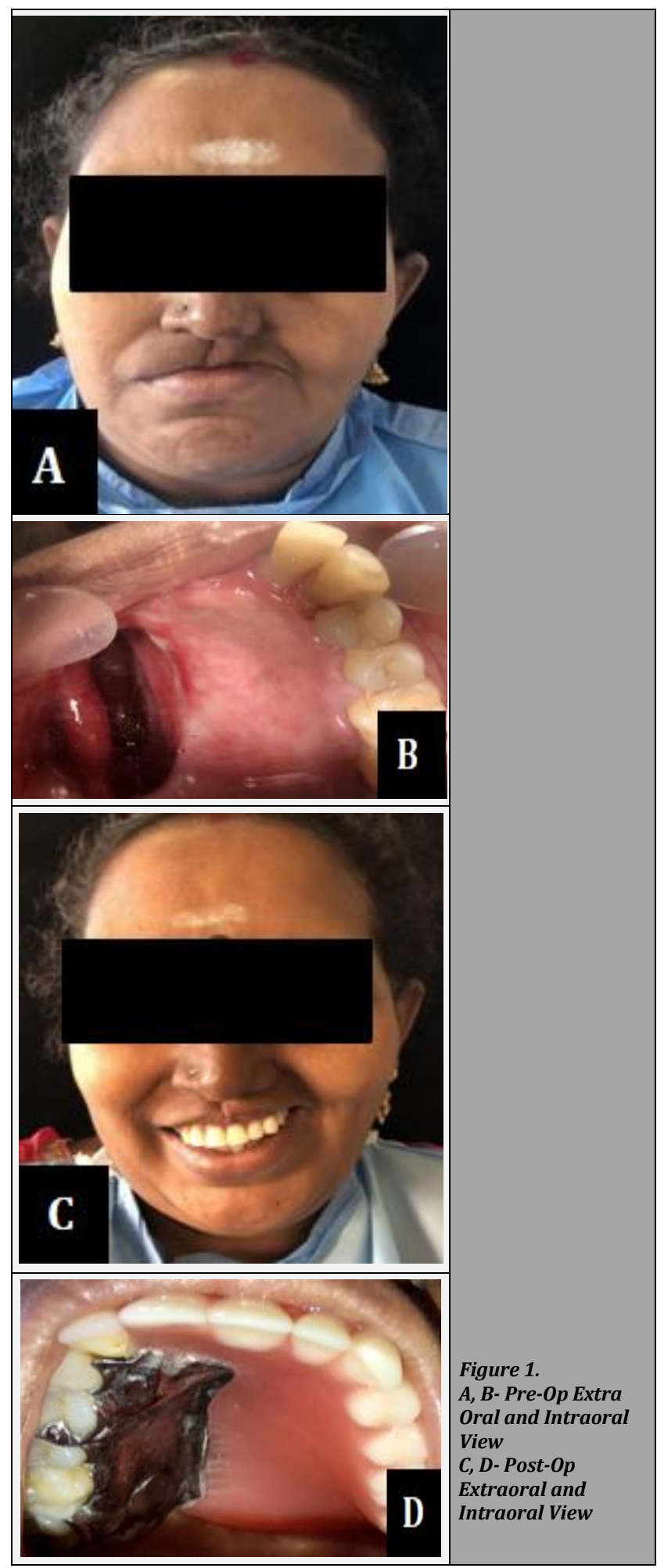

Weber Ferguson approach with sub ciliary extension was implemented, excising the alveolus in relation to $11-28$ extending horizontally till the palatal midline, and superiorly, all the antral walls including the orbital floor and rim was excised, preserving the orbital contents.

Following surgery, she received $66 \mathrm{GY}$ of radiotherapy in 33 fractions ( 5 fractions / week), single fraction daily for 6.5 weeks. Extra-oral examination revealed enophthalmos of the left eye, collapsed mid-face and surgical scar commencing at the philtrum, extending laterally around the base of the nose, superiorly along the nasofacial groove till the medial canthus, and laterally running within the skin crease below the ciliary line till the lateral canthus (Figure 1A). Intra-oral examination revealed partially dentate maxillary arch and left class III-b maxillectomydefect ${ }^{11}$ (Figure 1B).

\section{Diagnosis}

Squamous cell carcinoma involving the left maxilla.

\section{Discussion of Management}

For the prosthetic rehabilitation of the post-surgical defect, a single-piece open-hollow bulb definitive obturator prosthesis was planned and delivered to the patient (Figure 1C, 1D) (described under procedure for definitive obturator fabrication).

\section{PRESENTATION OF CASE 2}

A 64-year-old male patient was referred 10 days after surgery with a Ryle's tube for the fabrication of a delayed surgical obturator. He underwent infrastructure maxillectomy on the right side, superiorly involving the alveolar process of the maxilla relation to 21 - 18 , extending horizontally till the palatal midline. Extra-oral examination revealed collapsed right mid-face (Figure 2A). Intra-oral examination revealed partially dentate maxillary arch and right class II-b maxillectomy defect ${ }^{11}$ (Figure 2B).

\section{Diagnosis}

Rhino cerebral mucormycosis involving the right maxilla.

\section{Discussion of Management}

The patient was given a delayed surgical obturator for 20 days, followed by removal of the Ryle's tube and initiation of oral feeding. After 20 days, an interim obturator was fabricated and inserted for a period of 2 months until satisfactory healing of the surgical defect. On completion of healing, a single openhollow bulb definitive obturator prosthesis was delivered (Figure 2C, 2D) (described under procedure for definitive obturator fabrication). 


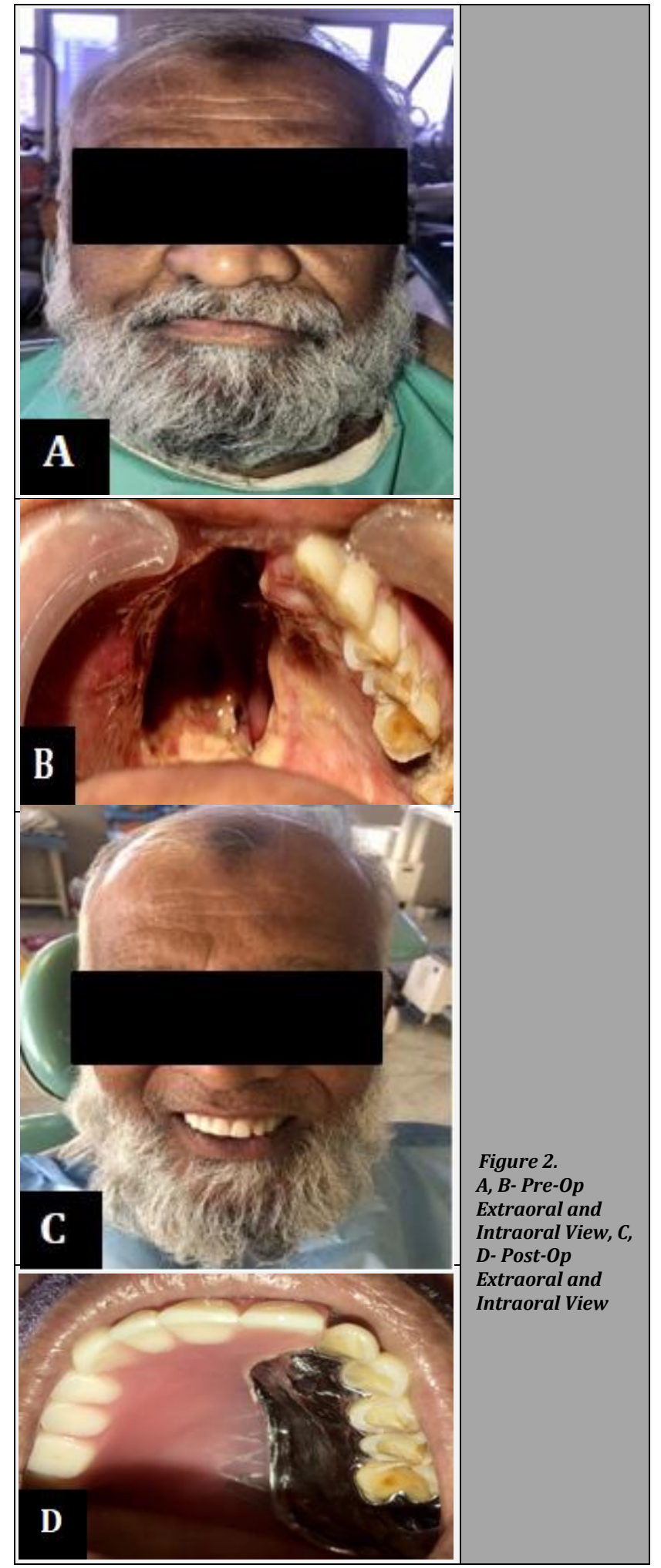

\section{PRESENTATION OF CASE 3}

A 45-year-old female patient was referred for the fabrication of immediate surgical obturator. She also reported difficulty during speech, mastication and deglutition. Extra-oral examination revealed no noticeable facial deformity. Intraoral examination revealed a swelling extending $4.5 \mathrm{cms}$ antero-posteriorly and $2.5 \mathrm{cms}$ mediolaterally on the palatal aspect of the left maxilla.

\section{Diagnosis}

Adenoid cystic carcinoma involving the left maxilla.
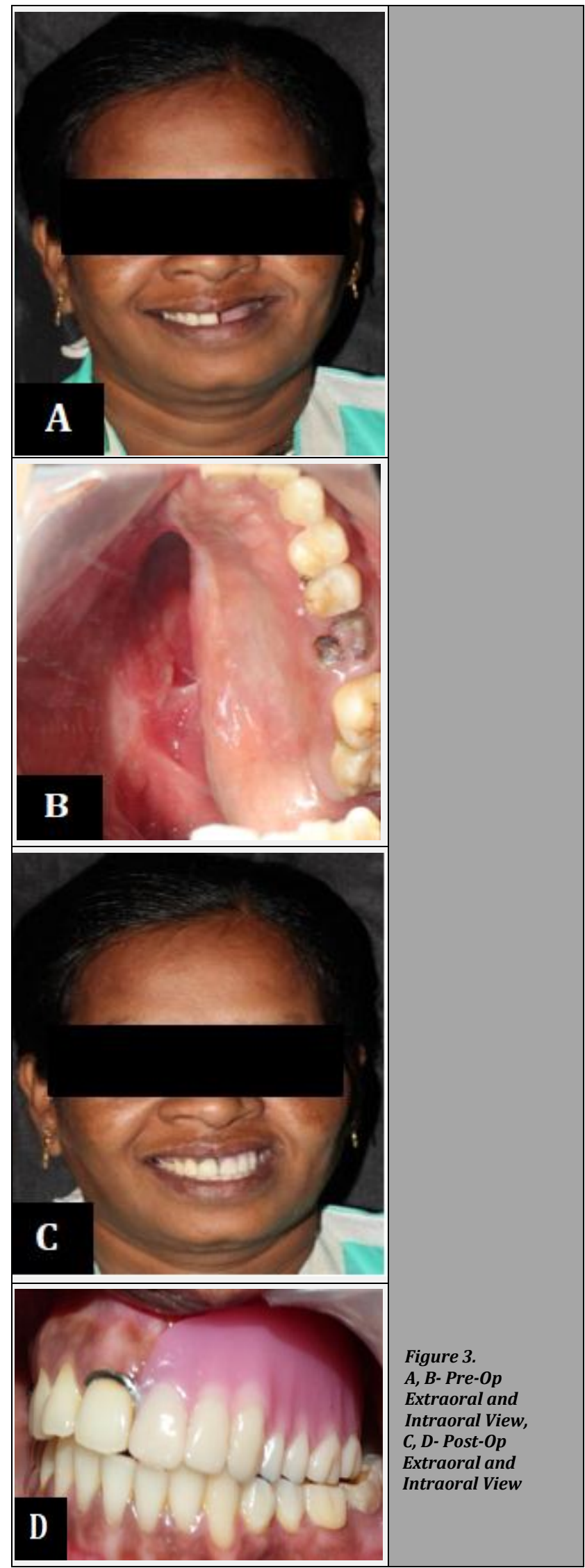


\section{Discussion of Management}

A pre-surgical alginate impression (Algitex, DPI, Mumbai, India) was made and type III dental stone cast (Goldstone, Gujarat, India) was prepared to mark the anticipated line of surgical resection. The outlined cast was verified with the surgeon and used for the fabrication of an immediate surgical obturator. The fabricated obturator was inserted by the surgeon immediately after surgery to protect the surgical site and prevent infection. Subtotal maxillectomy was performed on the patient. Weber Ferguson approach with sub ciliary extension was implemented, excising the alveolar process in relation to 21 - 28 extending horizontally till the palatal midline, and superiorly, all the antral walls including the orbital rim was excised, preserving the orbit. The patient reported back after a week for further management. Postsurgery, extra-oral examination revealed enophthalmos and diplopia in the left eye, collapsed mid-face and surgical scar commencing at the philtrum, extending laterally around the base of the nose, superiorly along the nasofacial groove till the medial canthus, and laterally running within the skin crease below the ciliary line till the lateral canthus (Figure 3A). Intraoral examination revealed partially dentate maxillary arch and left class III-b maxillectomydefect ${ }^{11}$ (Figure 3B). The patient was rehabilitated with an interim obturator for a period of 3 months until satisfactory healing of the surgical defect. On completion of healing, a single open-hollow bulb definitive obturator prosthesis was delivered (Figure 3C, 3D) (described under procedure for definitive obturator fabrication).

\section{PROCEDURE FOR DEFINITIVE OBTURATOR FABRICATION}

Maxillary and mandibular diagnostic impressions were made using alginate (Algitex, DPI, Mumbai, India), followed by fabrication of casts (type II dental stone, Goldstone, Gujarat, India) to be surveyed for cast partial framework design. Mouth preparations were done in accordance to the finalised framework design and maxillary polyvinyl siloxane (Zhermack, Elite $\mathrm{Hd}+$, Italy) impressions were made. These impressions were used for cast partial fabrication. The frameworks were tried intraorally to evaluate the fit. Special trays were fabricated using maxillary diagnostic casts, and full arch polyvinyl siloxane impressions were made, followed by preparation of master casts. Plaster pillars (Goldstone, Gujarat, India) were constructed in the defect region of the master casts, leaving 3 - $4 \mathrm{~mm}$ peripheral clearance, flushing at the height of remaining palatal vault, to create hollow-bulb in the obturators. The frameworks were then seated on the casts and connected to the fabricated record bases and occlusal rims. Face-bow transfer was done, jaw relations were recorded and mounted on semi-adjustable articulator. Teeth arrangements were done in accordance to the maxillary and mandibular remaining natural teeth, and verified for occlusion, phonetics and aesthetics. The obturators were processed using heat-cure acrylic resin (DPI, heat cure, denture base material, Mumbai, India), finished and polished (Figure 4A, 4B).

The obturators were inserted, re-evaluated for fit, occlusion and comfort, and the required adjustments were made. Post-insertion instructions were given, and the patients were asked to report back after 24 hours for review. Followup reviews were conducted for all the patients at 72 hours, 7 days, 1 month, 3 and 6 months, and prosthesis maintenance protocol was reinstated.

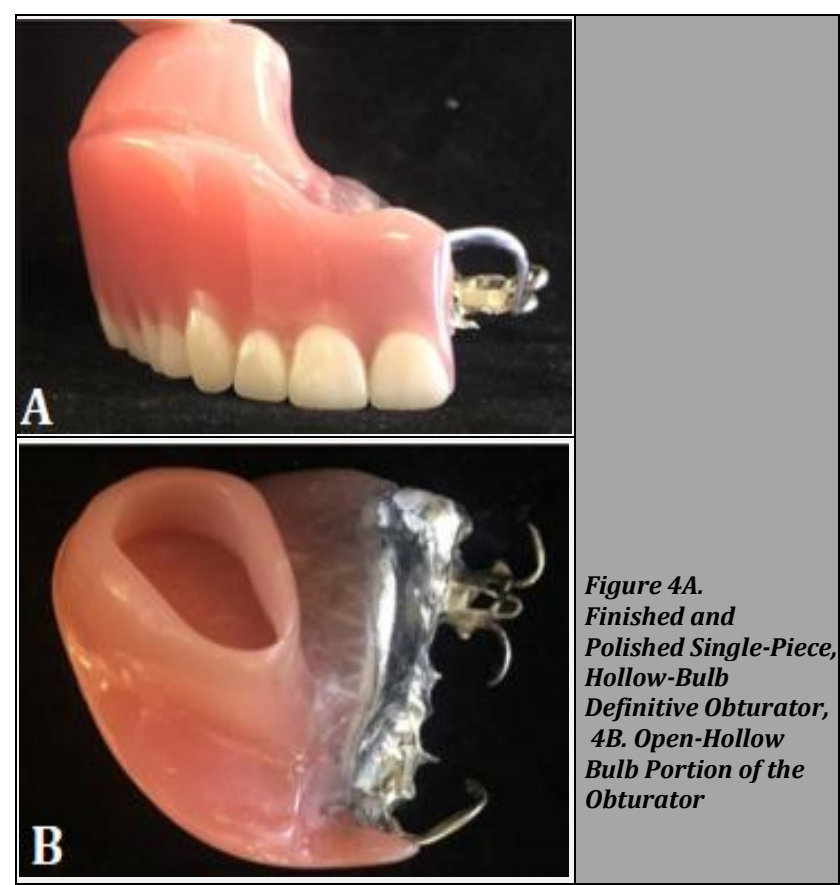

DISCUSSION

The definitive obturator is a maxillofacial prosthesis that replaces a portion, one or both the maxillae and the associated anatomy as a result of surgery or trauma. It is fabricated when tissue changes or recurrence of the tumour are unlikely and long-term prosthetic rehabilitation can be achieved. ${ }^{3,12}$ It incorporates a metal framework in its design which replicates the palate and supports the bulb portion of the obturator.

The primary objective of prosthetic rehabilitation of maxillectomy patients is to seal the surgical defect, thereby separating the oral from the nasal cavity, achieve acceptable aesthetics, support the orbital contents to prevent enophthalmos and diplopia, and support the soft tissues to restore the midfacialcontour. ${ }^{13}$ Thus, the obturator prosthesis prevents post-nasal drip, unintelligible articulation, deglutition difficulties, impaired mastication and unaesthetic appearance, and improves the patients psychological wellbeing. The removable nature of the prosthesis also enables the clinician to evaluate any future reoccurrences. In comparison to reconstructive plastic surgeries for the correction of maxillectomy defects, obturator prostheses are highly accepted treatment modality due to the ease of usage and maintenance. 12

The bulb portion of the obturator can be solid, openhollow or closed-hollow in design. The hollow design reduces prosthesis weight, improves retention and speech by providing resonance thus, improving patient comfort. ${ }^{14}$ Large maxillectomy defects are created as a result of aggressive lesions, especially those caused by carcinomas and mucormycosis. The duration of healing in such defects not only depends on the nature of the lesion, but also on the postoperative care. The reduction of prosthesis weight becomes essential when it is suspended without much remanent bone and posterior tooth support, as noticed in majority of 
maxillary resections. ${ }^{9}$ Thus, in such cases the hollow bulb design should be incorporated. Wu and Schaaf ${ }^{8}$ reported that the hollow bulb obturator reduced the overall prosthesis weight from $6.55 \%$ to $33.06 \%$, depending on the size of the maxillectomy defect. A lighter obturator improves the cantilever mechanics of suspension, prevents overtaxing of remaining supportive structures, and enhances retention. ${ }^{15}$ In this clinical report, the open-hollow bulb design was preferred over closed since it is lighter, easily adjustable and produces noticeably better articulation, as described by Oral et al. in their study. ${ }^{16}$ The plaster pillar employed for fabrication of the open-hollow bulb portion of the definitive obturator was simple, cost effective and an efficient method, and prevented prosthesis over reduction as the extensions were predetermined. ${ }^{17}$

\section{CONCLUSIONS}

This case report highlights the importance of prosthetic rehabilitation, as a component in the interdisciplinary care of life-threatening conditions. The single-piece, open-hollow bulb definitive obturator prosthesis complements the medical and surgical care by enhancing the wellbeing of the patient in terms of aesthetics, function and psychological aspects. The awareness of this treatment option among the physicians and surgeons, and the commitment of the prosthodontists resulted in improved patient outcome.

Financial or other competing interests: None.

Disclosure forms provided by the authors are available with the full text of this article at jemds.com.

The authors thank Dr. Anbarasi, Associate Professor, Department of Oral medicine and Radiology and Dr.Elengkumaran, Reader, Department of Oral and Maxillofa-cial surgery, Sri Ramachandra Institute of Higher Education and Research, Chennai, for their guidance and assistance in manuscript writing.

The authors certify that patient consent forms have been obtained. The patients have given their consent for the use of their images and other clinical information that has been reported in the journal. The patients understand that their names and initials will not be published, and efforts will be made to conceal their identity, but anonymity cannot be guaranteed.

\section{REFERENCES}

[1] Irish J, Sandhu N, Simpson C, et al. Quality of life in patients with maxillectomy prostheses. Head \& Neck 2009;31(6):813-21.

[2] Rogers SN, Lowe D, McNally D, et al. Health-related quality of life after maxillectomy: a comparison between prosthetic obturation and free flap. J Oral MaxillofacSurg 2003;61(2):174-81.

[3] Ferro KJ, Morgano SM, Driscoll CF, et al. The glossary of prosthodontic terms: ninth edition. J Prosthet Dent 2017;117(5S):e1-105.

[4] Devlin H, Barker GR. Prosthetic rehabilitation of the edentulous patient requiring a partial maxillectomy. J Prosthet Dent 1992;67(2):223-7.

[5] Keyf F.Obturator prostheses for hemimaxillectomy patients. J Oral Rehabil2001;28(9):821-9.

[6] Ortegon SM, Martin JW, LewinJS. A hollow delayed surgical obturator for a bilateral subtotal maxillectomy patient: a clinical report. J Prosthet Dent 2008;99(1):148.

[7] Gürbüz A, Hasanreisoğlu U. Clinical comparison of different types of obturators constructed after maxillary resections. Ankara UnivHekimFakDerg 1990;17(1):1038.

[8] Wu YL, Schaaf NG. Comparison of weight reduction in different designs of solid and hollow obturator prostheses. J Prosthet Dent 1989;62(2):214-7.

[9] Beumer J, Curtis TA, Marunick MT. Maxillofacial rehabilitation: prosthodontic and surgical considerations.IshiyakuEuroamerica 1996.

[10] Winkler S. Essentials of complete denture prosthodontics. St. Louis: Mosby Year Book1988.

[11] Brown JS, Shaw RJ. Reconstruction of the maxilla and midface: introducing a new classification. Lancet Oncol 2010;11(10):1001-8.

[12] Patil PG, Patil SP. A hollow definitive obturator fabrication technique for management of partial maxillectomy. J AdvProsthodont 2012;4(4):248-53.

[13] Wang RR. Sectional prosthesis for total maxillectomy patients: a clinical report. J Prosthet Dent 1997;78(3):241-4.

[14] Kamble VP, Desai RG, Arabbi KC. An innovative technique for fabrication of hollow bulb obturator: a case report. Unique J Med Dent Res 2013;1:42-5.

[15] Antoniou DV, ToljanicJA, Graham L.Obturator prosthesis retention for edentulous patients with large palatal defects: a clinical report. J Prosthet Dent 1996;76(3):2279.

[16] Oral K, Aramany MA, McWilliams BJ. Speech intelligibility with the buccal flange obturator. J Prosthet Dent 1979;41(3):323-8.

[17] Maheshwaran KS, Mohamed K, Subhiksha R, et al. A simplified novel approach in the fabrication of an interim hollow bulb obturator. Int J Dent Health Sci2017;4(5):1227-32. 